\title{
Brief Mindfulness Intervention for Emotional Distress, Resilience, and Compassion in Family Physicians During COVID-19: A Pilot Study
}

Ruth Nutting, PhD, LCMFT | Samuel Ofei-Dodoo, PhD, MPA, MA | Katherine Rose-Borcherding, DO | Grace Strella, MD

PRiMER. 2022;6:3.

Published: 2/14/2022 | DOI: 10.22454/PRiMER.2022.746202

\section{Abstract}

Background and Objective: There are high rates of professional burnout among family physicians and trainees. We undertook this study to investigate whether a brief mindfulness intervention could help manage burnout and improve well-being among family physicians in a residency program.

Methods: A total of 21 family physicians participated in a brief, 8-week mindfulness program. We used a single-sample, pre/post design at a Midwestern family medicine residency program. At two points in time (baseline and postintervention), participants completed an online survey measuring burnout, depression, anxiety, stress, perceived resilience, and compassion. We used linear mixed models to estimate the effect of the intervention on the outcome measures.

Results: Participants had improvements after the 8-week intervention. At postintervention, they had significantly better scores on anxiety $(P<.004)$, stress $(P<.001)$, perceived resilience $(P<.001)$, and compassion $(P<.001)$. There were no significant changes on the personal accomplishment, emotional exhaustion, and depersonalization subscales of either the abbreviated Maslach Burnout Inventory or the depression subscale of the Depression Anxiety Stress Scales-21.

Conclusion: This brief mindfulness program was associated with significant reduction in the scores of anxiety and stress as well as significant improvement in perceived resilience and compassion scores. Brief mindfulness interventions may be a convenient and effective approach to support and improve health and well-being among family physicians.

\section{Introduction}

Physicians are at higher risk for burnout than the average US adult. ${ }^{1,2}$ In 2020 , approximately $42 \%$ of physicians reported at least one manifestation of burnout, with family physicians ranking seventh of 30 specialties, at $47 \%{ }^{3}$ Burnout is characterized by long-term exposure to chronic job stressors that results in overwhelming exhaustion, depersonalization/cynicism, and decreased sense of personal accomplishment. ${ }^{4,5}$ Burnout has been attributed to organizational characteristics including declining reimbursements, heightened productivity expectations, and increased clerical burden. ${ }^{4}$ Personal characteristics such as perfectionism, self-judgement, 
and poor emotional regulation have also been accredited. ${ }^{5}$ Burnout among health professionals has been associated with decreased in quality of patient care, ${ }^{6-8}$ increased medical errors, ${ }^{9,10}$ decreased productivity, substance abuse, an elevated risk of depression, suicidal ideation, damage to relationship, $2,4,11,12$ and stronger intention of leaving the profession. ${ }^{6,13-15}$

As a mental state achieved by focusing attention on the present moment in a nonjudgmental way, ${ }^{16-18}$ mindfulness has been used to improve well-being, especially in health care since the 1970s in the form of an 8-week Mindfulness-Based Stress Reduction (MBSR) program. ${ }^{5}$ Literature indicates that mindfulness interventions help enhance coping skills and mitigate work-related distress by reducing symptoms of poor mental health, and increasing compassion and positive communication skills. ${ }^{5,14,16,17,19-28}$ However, there are challenges to implementing a traditional MBSR program, particularly within a family medicine residency program, as the 8-week program requires weekly 2.5-hour meetings, a 1-day retreat, and 45 minutes of daily homework. $^{7}$

We studied the impact of an intervention (75-minute sessions of weekly brief mindfulness for 8 weeks) on burnout, depression, anxiety, stress, resilience, and compassion among family physicians at a Midwestern family medicine residency program. We hypothesized that the physicians would have improved scores on the validated measures of burnout, depression, anxiety, stress, resilience, and compassion at the end of the 8-week intervention.

\section{Methods}

This study utilized a single-sample, nonexperimental design comparing pre- and postintervention scores on validated outcome measures between November 27, 2020 and January 29, 2021. We recruited 21 family physicians associated with the University of Kansas School of Medicine-Wichita (KUSM-W) Family Medicine Residency Program. We utilized email invitations and word of mouth to recruit participants. The study coordinator conducted telephone screening interview with volunteers who expressed interest. Those eligible were enrolled upon

receipt of a signed consent form. Participation was voluntary. The KUSM-W Institutional Review Board approved the study protocol for human subject research. The study utilized an 8-week brief mindfulness program led by trained and certified MBSR instructors who were not affiliated with our residence program. Each weekly session lasted 75-minutes. Topics covered included emotional intelligence, awareness, community building, communication, and stress resilience. Activities included mindfulness-meditation, body scans, and deep breathing. Participants were encouraged to practice mindfulness-skills received during the formal sessions for 20-minutes per day on their own.

Participants completed two online assessment surveys during the study: at baseline and immediately after the 8-week intervention period. Each survey consisted of the same four validated measures: the abbreviated Maslach Burnout Inventory (MBI-9), ${ }^{29-32}$ the Depression Anxiety Stress Scales-21 (DASS-21), ${ }^{33-35}$ the 14-item Resilience Scale (RS-14), ${ }^{36,37}$ and the Santa Clara Brief Compassion Scale (SCBC). ${ }^{38}$ We used these validated measures because we were interested in assessing the emotional state, resilience, and compassion of the participants. All the measures have been used in previous studies with a medical education population. ${ }^{2,11,12,31,40}$ The baseline survey also included demographic questions (Table 1). Linear mixed models were used to estimate effect of the brief mindfulness-intervention on the outcome variables of MBI-9, DASS-21, RES-14, and SCBC while adjusting for correlation due to repeated observations on each participant over the 8-week period. All analyses were two-sided with a of .05. 


\section{Participant Characteristics}

All 21 participants completed the baseline survey and 18 (86\%) provided data in the postintervention survey. All 18 participants completed the intervention and the recommended 20-minutes per day mindfulness-skills. The average age of participants was 32.9 years $(S D=9.3) ; 52.4 \%$ were male, and $76.2 \%$ were resident physicians (Table 1).

\section{Burnout}

As shown in Table 2, participants' baseline scores on MBI-9 were low, and showed no significant change.

\section{Depression, Anxiety, Stress, Resilience, and Compassion}

As Table 2 shows, the participants reported significant improvement in anxiety $(P<.01)$ and stress $(P<.01)$ DASS-21 scores after the intervention. Participants also had a significant improvement in perceived resilience scores $(P<.01)$ and in compassion scores postintervention $(P<.01)$.

\section{Discussion}

Our findings suggest that a brief mindfulness intervention may help support well-being and improve compassion among family physicians in residency programs. After 8 weeks of a brief mindfulness program, we found significant reductions in measures of anxiety and stress as well as improvements in resilience and compassion. These findings correlate with previous findings of health care professionals in diverse work environments. ${ }^{12,39}$ The beneficial findings are particularly noteworthy given that the brief intervention was given during the COVID-19 pandemic when many family physicians reported higher levels of stress and anxiety. ${ }^{40}$ While we believe that the effects would have been greater if the intervention had been the full MBSR program, the brief intervention may be enough to prepare the participants to continue mindfulness practices long-term. The observed lack of significant change in the emotional exhaustion, depersonalization, and personal accomplishment scores after the intervention may reflect the small sample size, and the participants' low burnout scores at the time of the study.

The significant positive improvement on perceived resilience among the participants is important in developing a workforce of family physicians who can maintain equilibrium in the face of stress and perceived risks. 2,12,28,41,42 Exposure to stress in health care setting is inevitable, but our findings suggest that this intervention is effective in helping physicians thrive even in stressful and challenging situations. Further, after the 8-week brief intervention, there was a significant improvement in compassion scores. Enhanced compassion and communication skills are key pillars of improving patients' related health care experiences and outcomes. ${ }^{19-25}$ Even with the modest improvement on the outcome measures, the findings suggest that the brief mindfulness intervention program likely provided the participants with wellness skills that can be used to improve their well-being. Thus, the brief mindfulness intervention program has a personal and/or clinical significance to the participants and the residence program.

A further step would be to implement similar mindfulness interventions in several residency programs, including those in other specialties, to determine how outcomes compare within and among different specialties. Outcome measures should be expanded to include the potential impact on patient care factors such as physician-patient relationships, health outcomes for patients, and cost savings to health care systems. Enhanced efforts should be made to incorporate long-term follow-up of the program's impact.

There were limitations to the study, given that it was conducted in a single residency program and had a small sample size. The nonexperimental nature of the study makes it difficult to infer causation and reduces generalizability as there is no way to know if the improvements were direct effects of the MBSR intervention. A 
randomized controlled trial is warranted to confirm the promising findings. Due to the size and composition of the program, we could not report findings based on career status nor race/ethnicity without compromising anonymity. Results of a prior study have shown that the level of emotional distress was significantly higher among residents than faculty. ${ }^{31}$

\section{Conclusion}

Our findings suggest that a brief mindfulness intervention of weekly 75-minute sessions over 8 weeks might be a way to improve resilience and compassion as well as reduce anxiety and stress among family physicians. Additional research is needed to confirm and expand on these findings.

\section{Tables and Figures}

Table 1: Participants' Characteristics at Baseline ( $\mathrm{N}=21)$

\begin{tabular}{|c|c|}
\hline Characteristics & All Respondents \\
\hline \multicolumn{2}{|l|}{ Biological Sex at Birth, n (\%) } \\
\hline Male & $11(52.4)$ \\
\hline \multicolumn{2}{|l|}{ Age, in Years } \\
\hline Mean (SD) & $32.9(9.3)$ \\
\hline Minimum & 27 \\
\hline Maximum & 62 \\
\hline \multicolumn{2}{|l|}{ Career Status, n (\%) } \\
\hline Full-time faculty & $5(23.8)$ \\
\hline Second-year residents & $5(31.3)$ \\
\hline Third-year residents & $5(31.3)$ \\
\hline
\end{tabular}


Table 2: Outcome Scores at Baseline and Postintervention

\begin{tabular}{|c|c|c|c|c|c|}
\hline \multirow[b]{2}{*}{ Subscale (Possible Range) } & \multicolumn{2}{|c|}{ Time Pointa } & \multirow[b]{2}{*}{$\beta$} & \multirow[b]{2}{*}{$\stackrel{P}{\text { Value }^{b}}$} & \multirow[b]{2}{*}{$\begin{array}{c}\text { Mean Difference } \\
(95 \% \mathrm{Cl})\end{array}$} \\
\hline & Baseline ( $\mathrm{N}=21)$ & $\begin{array}{l}\text { Post MBSR } \\
\text { Session }(N=18)\end{array}$ & & & \\
\hline MBI-9 Depersonalization (0-18) & $5.6(3.6-7.6)$ & $5.0(3.2-6.8)$ & 0.64 & .642 & $-1.22(-4.05$ to 1.60$)$ \\
\hline $\begin{array}{l}\text { MBI-9 Personal Accomplishment } \\
(0-18)\end{array}$ & $14.2(13.0-15.3)$ & $14.9(13.6-16.2)$ & -0.76 & .398 & $0.44(-1.55-2.44)$ \\
\hline DASS-21 Anxiety (0-21) & $3.5(1.9-5.1)$ & $1.0(0.6-1.5)$ & 2.52 & .004 & $-2.44(-4.25$ to -0.64$)$ \\
\hline DASS-21 Stress (0-21) & $7.3(5.4-9.3)$ & $2.8(2.1-3.4)$ & 4.56 & .001 & $-4.56(6.68$ to -2.43$)$ \\
\hline RS-14 (14-98) & $79.4(63.9-84.9)$ & $92.1(90.7-93.4)$ & -12.64 & .001 & 10.05 (2.88 to 17.22$)$ \\
\hline
\end{tabular}

Abbreviations: MBI-9, Maslach Burnout Inventory; DASS-21, Depression Anxiety Stress Scales-21; RS-14, 14-item Resilience Scale; SCBC, Santa Clara Brief Compassion Scale.

On MBI-9, higher scores on the Emotional Exhaustion and Depersonalization subscales, and lower scores on the Personal Accomplishment subscale indicate greater burnout. On the DASS-21 subscales, higher scores indicate greater levels of that emotional state. On the RS-14, higher scores indicating greater resilience.

On the SCBC, higher scores indicate greater compassion.

a Values shown are mean score $(95 \% \mathrm{Cl})$.

b $P$ values were calculated with the linear mixed effects models and denote the significance of $\beta$ coefficients.

\section{Acknowledgments}

Presentations: The findings from this study were presented in May 2021 at the Society of Teachers of Family Medicine Annual Spring Conference (virtual).

Funding Statement: Funding for this study was provided by the Ascension Via Christi Philanthropy Foundation.

\section{Corresponding Author}

Samuel Ofei-Dodoo, PhD, MPA, MA

Department of Family and Community Medicine, University of Kansas School of Medicine-Wichita, $1010 \mathrm{~N}$ Kansas St, Wichita, KS 67214. 316-293-1879. Fax: 316-293-2696.

sofeidodoo@kumc.edu

\section{Author Affiliations}

Ruth Nutting, PhD, LCMFT - University of Kansas School of Medicine-Wichita, Department of Family and Community Medicine, Wichita, KS

Samuel Ofei-Dodoo, PhD, MPA, MA - Department of Family and Community Medicine, University of Kansas School of Medicine-Wichita, Wichita, KS

Katherine Rose-Borcherding, DO - University of Kansas School of Medicine-Wichita, Department of Family and Community Medicine, Wichita, KS

Grace Strella, MD - University of Kansas School of Medicine-Wichita, Department of Family and Community Medicine, Wichita, KS

\section{References}

1. Shanafelt TD, West CP, Sinsky C, et al. Changes in burnout and satisfaction with work-life integration in physicians and the general US working population between 2011 and 2017. Mayo Clin Proc.

2019;94(9):1681-1694. doi:10.1016/j.mayocp.2018.10.023 
2. Ofei-Dodoo S, Mullen R, Pasternak A, et al. Loneliness, burnout, and other types of emotional distress among family medicine physicians: results from a national survey. J Am Board Fam Med.

2021;34(3):531-541. doi:10.3122/jabfm.2021.03.200566

3. Kane L. Death by 1000 cuts: Medscape national physician burnout and suicide report 2021: the generational divide. Medscape. Published January 15, 2020. Accessed April 28, 2020.

https://www.medscape.com/slideshow/2021-lifestyle-burnout-6013456\#2.

4. Maslach C. Job burnout. Curr Dir Psychol Sci. 2003;12(5):189-192. doi:10.1111/1467-8721.01258

5. Shanafelt TD, Noseworthy JH. Executive leadership and physician well-being: nine organizational strategies to promote engagement and reduce burnout. Mayo Clin Proc. 2017;92(1):129-146. doi:10.1016/j.mayocp.2016.10.004

6. Dewa CS, Loong D, Bonato S, Trojanowski L. The relationship between physician burnout and quality of healthcare in terms of safety and acceptability: a systematic review. BMJ Open. 2017;7(6):e015141. doi:10.1136/bmjopen-2016-015141

7. Weigl M, Schneider A, Hoffmann F, Angerer P. Work stress, burnout, and perceived quality of care: a cross-sectional study among hospital pediatricians. Eur J Pediatr. 2015;174(9):1237-1246. doi:10.1007/s00431-015-2529-1

8. Shirom A, Nirel N, Vinokur AD. Overload, autonomy, and burnout as predictors of physicians' quality of care. J Occup Health Psychol. 2006;11(4):328-342. doi:10.1037/1076-8998.11.4.328

9. Hayashino Y, Utsugi-Ozaki M, Feldman MD, Fukuhara S. Hope modified the association between distress and incidence of self-perceived medical errors among practicing physicians: prospective cohort study. PLoS One. 2012;7(4):e35585. doi:10.1371/journal.pone.0035585

10. Wen J, Cheng Y, Hu X, Yuan P, Hao T, Shi Y. Workload, burnout, and medical mistakes among physicians in China: A cross-sectional study. Biosci Trends. 2016;10(1):27-33. doi:10.5582/bst.2015.01175

11. Ofei-Dodoo S, Kellerman R, Gilchrist K, Casey EM. Burnout and quality of life among active member physicians of the Medical Society of Sedgwick County. Kans J Med. 2019;12(2):33-39. doi:10.17161/kjm.v12i2.11701

12. Ofei-Dodoo S, Cleland-Leighton A, Nilsen K, Cloward JL, Casey E. Impact of a mindfulness-based, workplace group yoga intervention on burnout, self-care, and compassion in health care professionals: a pilot study. J Occup Environ Med. 2020;62(8):581-587. doi:10.1097/JOM.0000000000001892

13. Gregory ST, Menser T, Gregory BT. An organizational intervention to reduce physician burnout. J Healthc Manag. 2018;63(5):338-352. doi:10.1097/JHM-D-16-00037

14. Linzer M, Poplau S, Grossman E, et al. A cluster randomized trial of interventions to improve work conditions and clinician burnout in primary care: results from the Healthy Work Place (HWP) study. J Gen Intern Med. 2015;30(8):1105-1111. doi:10.1007/s11606-015-3235-4

15. Maslach C. Job burnout. Curr Dir Psychol Sci. 2003;12(5):189-192. doi:10.1111/1467-8721.01258

16. Beach MC, Roter D, Korthuis PT, et al. A multicenter study of physician mindfulness and health care quality. Ann Fam Med. 2013;11(5):421-428. doi:10.1370/afm.1507

17. Valley $M$, Stallones $L$. A thematic analysis of health care workers' adoption of mindfulness practices. Workplace Health Saf. 2018;66(11):538-544. doi:10.1177/2165079918771991

18. Kabat-Zinn J. Mindfulness-based interventions in context: past, present and future. Clin Psychol. 2003;10(2):144-156.

19. de Vibe M, Solhaug I, Rosenvinge JH, Tyssen R, Hanley A, Garland E. Six-year positive effects of a mindfulness-based intervention on mindfulness, coping and well-being in medical and psychology students; Results from a randomized controlled trial. PLoS One. 2018;13(4):e0196053.

doi:10.1371/journal.pone.0196053

20. McConville J, McAleer R, Hahne A. Mindfulness training for health profession students-the effect of mindfulness training on psychological well-being, learning and clinical performance of health professional students: a systematic review of randomized and non-randomized controlled trials. Explore 
(NY). 2017;13(1):26-45. doi:10.1016/j.explore.2016.10.002

21. Fox S, Lydon S, Byrne D, Madden C, Connolly F, O'Connor P. A systematic review of interventions to foster physician resilience. Postgrad Med J. 2018;94(1109):162-170. doi:10.1136/postgradmedj-2017-135212

22. Thirioux B, Birault F, Jaafari N. Empathy is a protective factor of burnout in physicians: new neurophenomenological hypotheses regarding empathy and sympathy in care relationship. Front Psychol. 2016;7(763):763. doi:10.3389/fpsyg.2016.00763

23. Amutio-Kareaga A, García-Campayo J, Delgado LC, Hermosilla D, Martínez-Taboada C. Improving communication between physicians and their patients through mindfulness and compassion-based strategies: a narrative review. J Clin Med. 2017;6(3):33. doi:10.3390/jcm6030033

24. Ireland MJ, Clough B, Gill K, Langan F, O'Connor A, Spencer L. A randomized controlled trial of mindfulness to reduce stress and burnout among intern medical practitioners. Med Teach. 2017;39(4):409-414. doi:10.1080/0142159X.2017.1294749

25. O'Mahony S, Gerhart J, Abrams I, et al. A multimodal mindfulness training to address mental health symptoms in providers who care for and interact with children in relation to end-of-life care. Am J Hosp Palliat Care. 2017;34(9):838-843. doi:10.1177/1049909116660688

26. Slater PJ, Edwards RM, Badat AA. Evaluation of a staff well-being program in a pediatric oncology, hematology, and palliative care services group. J Healthc Leadersh. 2018;10:67-85. doi:10.2147/JHL.S176848

27. Goyal M, Singh S, Sibinga EM, et al. Meditation programs for psychological stress and well-being: a systematic review and meta-analysis. JAMA Intern Med. 2014;174(3):357-368. doi:10.1001/jamainternmed.2013.13018

28. Minichiello V, Hayer S, Gillespie B, Goss M, Barrett B. Developing a mindfulness skills-based training program for resident physicians. Fam Med. 2020;52(1):48-52. doi:10.22454/FamMed.2020.461348

29. McManus IC, Keeling A, Paice E. Stress, burnout and doctors' attitudes to work are determined by personality and learning style: a twelve year longitudinal study of UK medical graduates. BMC Med. 2004;2(29):29. doi:10.1186/1741-7015-2-29

30. Maslach C, Leiter MP. Early predictors of job burnout and engagement. J Appl Psychol. 2008;93(3):498-512. doi:10.1037/0021-9010.93.3.498

31. Ofei-Dodoo S, Callaway P, Engels K. Prevalence and etiology of burnout in a community-based graduate medical education system: a mixed-methods study. Fam Med. 2019;51(9):766-771. doi:10.22454/FamMed.2019.431489

32. Ofei-Dodoo S, Scripter C, Kellerman R. Job satisfaction and burnout among nonclinical workers in a medical education center. Fam Med. 2018;50(3):223-227. doi:10.22454/FamMed.2018.473306

33. Lovibond SH, Lovibond PF. Manual for the Depression Anxiety Stress Scales. 2nd ed. Sydney, Australia: Psychology Foundation; 1995.

34. Gomez F. A guide to the depression, anxiety, and stress scale (DASS 21). Accessed February 23, 2020. https://jeanmartainnaturopath.com.au/wp-content/uploads/2016/10/Dass21.pdf

35. Osman A, Wong JL, Bagge CL, Freedenthal S, Gutierrez PM, Lozano G. The Depression Anxiety Stress Scales-21 (DASS-21): further examination of dimensions, scale reliability, and correlates. J Clin Psychol. 2012;68(12):1322-1338. doi:10.1002/jclp.21908

36. Wagnild G. The Resilience Scale User's Guide for the US English Version of the Resilience Scale and the 14-Item Resilience Scale (RS-14). The Resilience Center; 2009.

37. Miroševič Š, Klemenc-Ketiš Z, Selič P. The 14-item Resilience scale as a potential screening tool for depression/anxiety and quality of life assessment: a systematic review of current research. Fam Pract. 2019;36(3):262-268. doi:10.1093/fampra/cmy081

38. Hwang JY, Plante T, Lackey K. The development of the Santa Clara Brief Compassion Scale: an abbreviation of Sprecher and Fehr's Compassionate Love Scale. Pastoral Psychol. 2008;56(4):421-428. doi:10.1007/s11089-008-0117-2 
39. Fortney L, Luchterhand C, Zakletskaia L, Zgierska A, Rakel D. Abbreviated mindfulness intervention for job satisfaction, quality of life, and compassion in primary care clinicians: a pilot study. Ann Fam Med. 2013;11(5):412-420. doi:10.1370/afm.1511

40. Ofei-Dodoo S, Loo-Gross C, Kellerman R. Burnout, Depression, Anxiety, and Stress Among Family Physicians in Kansas Responding to the COVID-19 Pandemic. J Am Board Fam Med. 2021;34(3):522-530. doi:10.3122/jabfm.2021.03.200523

41. Tarantino B, Earley M, Audia D, D'Adamo C, Berman B. Qualitative and quantitative evaluation of a pilot integrative coping and resiliency program for healthcare professionals. Explore (NY). 2013;9(1):44-47. doi:10.1016/j.explore.2012.10.002

42. Beckwith H. Building resilience in health care workers. Clin Teach. 2016;13(6):457-460. doi:10.1111/tct.12603

Copyright $(2022$ by the Society of Teachers of Family Medicine 